\title{
EXPLICIT ISOMORPHISMS OF REAL CLIFFORD ALGEBRAS
}

\author{
N. DEĞİRMENCİ AND Ş. KARAPAZAR \\ Received 13 March 2005; Revised 6 January 2006; Accepted 27 February 2006
}

It is well known that the Clifford algebra $\mathrm{Cl}_{p, q}$ associated to a nondegenerate quadratic form on $\mathbb{R}^{n}(n=p+q)$ is isomorphic to a matrix algebra $K(m)$ or direct sum $K(m) \oplus$ $K(m)$ of matrix algebras, where $K=\mathbb{R}, \mathbb{C}, \mathbb{M}$. On the other hand, there are no explicit expressions for these isomorphisms in literature. In this work, we give a method for the explicit construction of these isomorphisms.

Copyright @ 2006 Hindawi Publishing Corporation. All rights reserved.

\section{Preliminaries}

Let $F$ be a field and let $V$ be a finite-dimensional vector space over $F$ and $Q: V \rightarrow F$ a quadratic form on $V$. The Clifford algebra $\mathrm{Cl}(V, Q)$ is an associative algebra with unit 1 , which contains and is generated by $V$, with $v \cdot v=Q(v) \cdot 1$ for all $v \in V$. Formally, one can define the Clifford algebra $\mathrm{Cl}(V, Q)$ as follows.

Definition 1.1. The Clifford algebra $\mathrm{Cl}(V, Q)$ associated to a vector space $V$ over $F$ with quadratic form $Q$ can be defined as

$$
\mathrm{Cl}(V, Q)=\frac{T(V)}{I(Q)}
$$

where $T(V)$ is the tensor algebra $T(V)=F \oplus V \oplus(V \otimes V) \oplus \cdots$ and $I(Q)$ is the twosided ideal in $T(V)$ generated by elements $v \otimes v-Q(v) \cdot 1$.

Just like the tensor algebra and the exterior algebra, the Clifford algebra has the following universal property.

Theorem 1.2. Given an associative unital F-algebra $A$ (with unit 1) and a linear map $f: V \rightarrow A$ with $f(v) \cdot f(v)=Q(v) \cdot 1$ for all $v \in V$, then there is a unique homomorphism 
2 Explicit isomorphisms of real Clifford algebras

of algebras $f: \mathrm{Cl}(V, Q) \rightarrow A$ such that the following diagram commutes:

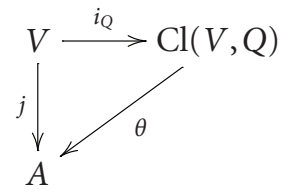

where $i_{Q}$ is natural inclusion. In particular, the algebra $\mathrm{Cl}(V, Q)$ together with the map $i_{Q}: V \rightarrow \mathrm{Cl}(V, Q)$ satisfying $i_{Q}(v) \cdot i_{Q}(v)=Q(v) \cdot 1$ is uniquely determined by this property up to isomorphism (see [3]).

If $Q=0$, one recovers precisely the definition of exterior algebra, so $\wedge(V)=\mathrm{Cl}(V, Q=$ $0)$.

For the realization of the Clifford algebra $\mathrm{Cl}(V, Q)$, the following lemma is useful.

Lemma 1.3. The structure map $i_{Q}: V \rightarrow \mathrm{Cl}(V, Q)$ is injective. Thus $V$ will be viewed as a subspace of $\mathrm{Cl}(V, Q)$. If $e_{1}, e_{2}, \ldots, e_{n}$ form a basis: for $V$, then the products

$$
e_{i_{1}} e_{i_{2}} \cdots e_{i_{k}}, \quad 1 \leq i_{1}<\cdots<i_{k} \leq n
$$

and 1 form a basis of the real vector space $\mathrm{Cl}(V, Q)($ see $[2,3])$.

We deal with the real vector spaces with nondegenerate quadratic form $Q$. Due to the Sylvester theorem, any nondegenerate quadratic form on $\mathbb{R}^{n}$ is equivalent to a quadratic form of type

$$
Q\left(x_{1}, x_{2}, \ldots, x_{n}\right)=x_{1}^{2}+x_{2}^{2}+\cdots+x_{p}^{2}-x_{p+1}^{2}-x_{p+2}^{2}-\cdots-x_{p+q}^{2}
$$

(see [1]). If $V=\mathbb{R}^{n}$ is a real vector space with the quadratic form $Q: \mathbb{R}^{n} \rightarrow \mathbb{R}, Q\left(x_{1}, x_{2}, \ldots\right.$, $\left.x_{n}\right)=x_{1}^{2}+x_{2}^{2}+\cdots+x_{p}^{2}-x_{p+1}^{2}-x_{p+2}^{2}-\cdots-x_{p+q}^{2}$, then the corresponding Clifford algebra $\mathrm{Cl}(V, Q)$ is denoted by $\mathrm{Cl}_{p, q}(n=p+q)$. Let $e_{1}, e_{2}, \ldots, e_{p}, \varepsilon_{1}, \varepsilon_{2}, \ldots, \varepsilon_{q}$ be a Sylvester basis for $\mathbb{R}^{n}$, then following relations hold: $e_{i}^{2}=1(1 \leq i \leq p), \varepsilon_{i}^{2}=-1(1 \leq i \leq q)$ and $e_{i} e_{j}=-e_{j} e_{i}, \varepsilon_{i} \varepsilon_{j}=-\varepsilon_{j} \varepsilon_{i}$ for $i \neq j$ and $e_{i} \varepsilon_{j}=-\varepsilon_{j} e_{i}$ for $1 \leq i \leq p, 1 \leq j \leq q$.

1.1. Calculations for some lower dimensions. Let $\Psi_{p, q}$ denote the isomorphism from the Clifford algebra $\mathrm{Cl}_{p, q}$ to the related matrix algebra and for $K=\mathbb{R}, \mathbb{C}$, or $\mathbb{U}$, we denote by $K(n)$ the algebra of $n \times n$-matrices with entries in $K$.

For $n=0, \mathrm{Cl}_{0,0} \cong \mathbb{R}$

For $n=1, \mathrm{Cl}_{0,1} \cong \mathbb{C}$ by $\Psi_{0,1}(e)=i$ and $\mathrm{Cl}_{1,0} \cong \mathbb{R} \oplus \mathbb{R}$ by $\Psi_{1,0}(e)=(-1,1)$

For $n=2$,

(i) the Clifford algebra $\mathrm{Cl}_{0,2}$ is isomorphic to the quaternion algebra $\mathbb{U}$ by th isomorphism $\Psi_{0,2}: \mathrm{Cl}_{0,2} \rightarrow \mathbb{H}, \Psi_{0,2}\left(e_{1}\right)=i, \Psi_{0,2}\left(e_{2}\right)=j$ and so $\Psi_{0,2}\left(e_{1} e_{2}\right)=k$;

(ii) the Clifford algebra $\mathrm{Cl}_{2,0}$ is isomorphic to the matrix algebra $\mathbb{R}(2)$ by the isomorphism $\Psi_{2,0}: \mathrm{Cl}_{2,0} \rightarrow \mathbb{R}(2), \Psi_{2,0}\left(e_{1}\right)=\left(\begin{array}{cc}1 & 0 \\ 0 & -1\end{array}\right), \Psi_{2,0}\left(e_{2}\right)=\left(\begin{array}{ll}0 & 1 \\ 1 & 0\end{array}\right)$ and so $\Psi_{2,0}\left(e_{1} e_{2}\right)=$ $\left(\begin{array}{cc}0 & 1 \\ -1 & 0\end{array}\right)$; 
(iii) the Clifford algebra $\mathrm{Cl}_{1,1}$ is isomorphic to the matrix algebra $\mathbb{R}(2)$ by the isomorphism $\Psi_{1,1}: \mathrm{Cl}_{1,1} \rightarrow \mathbb{R}(2), \Psi_{1,1}\left(e_{1}\right)=\left(\begin{array}{cc}1 & 0 \\ 0 & -1\end{array}\right), \Psi_{1,1}\left(\varepsilon_{1}\right)=\left(\begin{array}{cc}0 & 1 \\ -1 & 0\end{array}\right)$ and so $\Psi_{1,1}\left(e_{1} \varepsilon_{1}\right)=$ $\left(\begin{array}{ll}0 & 1 \\ 1 & 0\end{array}\right)$.

To determine $\mathrm{Cl}_{p, q}$ for higher values of $n=p+q$, the following proposition is useful.

Proposition 1.4. There are isomorphisms

$$
\begin{aligned}
& \mathrm{Cl}_{0, m+2} \cong \mathrm{Cl}_{m, 0} \otimes \mathrm{Cl}_{0,2}, \\
& \mathrm{Cl}_{m+2,0} \cong \mathrm{Cl}_{0, m} \otimes \mathrm{Cl}_{2,0}, \\
& \mathrm{Cl}_{p+1, q+1} \cong \mathrm{Cl}_{p, q} \otimes \mathrm{Cl}_{1,1} .
\end{aligned}
$$

(We note that ungraded tensor product is meant throughout the paper.)

The first isomorphism $\pi_{1}: \mathrm{Cl}_{0, m+2} \rightarrow \mathrm{Cl}_{m, 0} \otimes \mathrm{Cl}_{0,2}$ is given by

$$
\pi_{1}\left(e_{i}\right)= \begin{cases}\varepsilon_{i-2} \otimes e_{1} e_{2}, & \text { if } 3 \leq i \leq m+2, \\ 1 \otimes e_{i}, & \text { if } i=1 \text { and } i=2,\end{cases}
$$

the second isomorphism $\pi_{2}: \mathrm{Cl}_{m+2,0} \rightarrow \mathrm{Cl}_{0, m} \otimes \mathrm{Cl}_{2,0}$ can be given by

$$
\pi_{2}\left(\varepsilon_{i}\right)= \begin{cases}e_{i-2} \otimes \varepsilon_{1} \varepsilon_{2}, & \text { if } 3 \leq i \leq m+2, \\ 1 \otimes \varepsilon_{i}, & \text { if } i=1 \text { and } i=2\end{cases}
$$

and the third one $\pi_{3}: \mathrm{Cl}_{p+1, q+1} \rightarrow \mathrm{Cl}_{p, q} \otimes \mathrm{Cl}_{1,1}$ can be given by

$$
\begin{aligned}
& \pi_{3}\left(e_{i}\right)= \begin{cases}e_{i} \otimes e_{1} \varepsilon, & \text { if } 1 \leq i \leq p, \\
1 \otimes e_{1}, & \text { if } i=p+1,\end{cases} \\
& \pi_{3}\left(\varepsilon_{j}\right)= \begin{cases}\varepsilon_{j} \otimes e_{1} \varepsilon_{1}, & \text { if } 1 \leq j \leq q, \\
1 \otimes \varepsilon_{1}, & \text { if } j=q+1 .\end{cases}
\end{aligned}
$$

By applying the above isomorphisms recursively, it is possible to get isomorphisms of Clifford algebras, but to apply these isomorphisms, we need some further isomorphisms among the various real algebras.

Proposition 1.5. The following isomorphisms hold.

(i) $\mathbb{R}(m) \otimes K \cong K(m)$ by $\left[a_{i j}\right] \otimes k \mapsto\left[a_{i j} k\right]$ where $K=\mathbb{R}$, $\mathbb{C}$, or $\mathbb{H}$.

(ii) $\mathbb{R}(m) \otimes \mathbb{R}(n) \cong \mathbb{R}(m n)$ by $A \otimes B \mapsto\left[a_{i j} B\right]$ (this operation is called the Kronecker product of $A$ and $B)$, where $A=\left[a_{i j}\right]$.

(iii) $\mathbb{C} \otimes \mathbb{U} \cong \mathbb{C}(2)$. For this isomorphism, consider $\mathbb{U}$ as a $\mathbb{C}$ module under left scalar multiplication, and define an $\mathbb{R}$-bilinear map $\Psi: \mathbb{C} \times \mathbb{M} \rightarrow \mathrm{Hom}_{\mathbb{C}}(\mathbb{M}, \mathbb{H})$ by setting $\Psi_{z, q}(x)=z x \bar{q}$ and this extends (by the universal property of tensor product) to an $\mathbb{R}$-linear map $\Psi: \mathbb{C} \otimes \mathbb{U} \rightarrow \operatorname{Hom}_{\mathbb{C}}(\mathbb{M}, \mathbb{U}) \cong \mathbb{C}(2)$. This is an isomorphism (see [3]). The images of the basis elements $1 \otimes 1,1 \otimes i, 1 \otimes j, 1 \otimes k, i \otimes 1, i \otimes i, i \otimes j, i \otimes k$ of 
$\mathbb{C} \otimes \mathbb{U}$ under this isomorphism are as follows:

$$
\begin{aligned}
& \Psi_{1,1}=\left[\begin{array}{ll}
1 & 0 \\
0 & 1
\end{array}\right], \quad \Psi_{1, i}=\left[\begin{array}{cc}
-i & 0 \\
0 & i
\end{array}\right], \quad \Psi_{1, j}=\left[\begin{array}{cc}
0 & 1 \\
-1 & 0
\end{array}\right], \quad \Psi_{1, k}=\left[\begin{array}{cc}
0 & -i \\
-i & 0
\end{array}\right], \\
& \Psi_{i, 1}=\left[\begin{array}{cc}
i & 0 \\
0 & i
\end{array}\right], \quad \Psi_{i, i}=\left[\begin{array}{cc}
1 & 0 \\
0 & -1
\end{array}\right], \quad \Psi_{i, j}=\left[\begin{array}{cc}
0 & -i \\
i & 0
\end{array}\right], \quad \Psi_{i, k}=\left[\begin{array}{ll}
0 & 1 \\
1 & 0
\end{array}\right] \text {. }
\end{aligned}
$$

(iv) $\mathbb{U} \otimes \mathbb{U} \cong \mathbb{R}(4)$. For this isomorphism, consider the $\mathbb{R}$-bilinear map $\Psi: \mathbb{M} \times \mathbb{U} \rightarrow$ $\operatorname{Hom}_{\mathbb{R}}(\mathbb{H}, \mathbb{H}) \cong \mathbb{R}(4)$ given by $\Psi_{q_{1}, q_{2}}(x)=q_{1} x \overline{q_{2}}$. This map extends (by the universal property of tensor product) to an $\mathbb{R}$-linear map $\Psi: \mathbb{H} \otimes \mathbb{U} \rightarrow \operatorname{Hom}_{\mathbb{R}}(\mathbb{H}, \mathbb{H}) \cong \mathbb{R}(4)$. This is an isomorphism (see [3]). The images of the basis elements $1 \otimes 1,1 \otimes i, 1 \otimes j$, $1 \otimes k, i \otimes 1, i \otimes i, i \otimes j, i \otimes k, j \otimes 1, j \otimes i, j \otimes j, j \otimes k, k \otimes 1, k \otimes i, k \otimes j, k \otimes k$ of $\mathbb{H} \otimes \mathbb{U}$ under this isomorphism areas are as follows:

$$
\begin{aligned}
& \Phi_{1,1}=\left[\begin{array}{llll}
1 & 0 & 0 & 0 \\
0 & 1 & 0 & 0 \\
0 & 0 & 1 & 0 \\
0 & 0 & 0 & 1
\end{array}\right], \quad \Phi_{1, i}=\left[\begin{array}{cccc}
0 & 1 & 0 & 0 \\
-1 & 0 & 0 & 0 \\
0 & 0 & 0 & -1 \\
0 & 0 & 1 & 0
\end{array}\right], \quad \Phi_{1, j}=\left[\begin{array}{cccc}
0 & 0 & 1 & 0 \\
0 & 0 & 0 & 1 \\
-1 & 0 & 0 & 0 \\
0 & -1 & 0 & 0
\end{array}\right], \\
& \Phi_{1, k}=\left[\begin{array}{cccc}
0 & 0 & 0 & 1 \\
0 & 0 & -1 & 0 \\
0 & 1 & 0 & 0 \\
-1 & 0 & 0 & 0
\end{array}\right], \quad \Phi_{i, 1}=\left[\begin{array}{cccc}
0 & -1 & 0 & 0 \\
1 & 0 & 0 & 0 \\
0 & 0 & 0 & -1 \\
0 & 0 & 1 & 0
\end{array}\right], \quad \Phi_{i, i}=\left[\begin{array}{cccc}
1 & 0 & 0 & 0 \\
0 & 1 & 0 & 0 \\
0 & 0 & -1 & 0 \\
0 & 0 & 0 & -1
\end{array}\right], \\
& \Phi_{i, j}=\left[\begin{array}{cccc}
0 & 0 & 0 & -1 \\
0 & 0 & 1 & 0 \\
0 & 1 & 0 & 0 \\
-1 & 0 & 0 & 0
\end{array}\right], \quad \Phi_{i, k}=\left[\begin{array}{llll}
0 & 0 & 1 & 0 \\
0 & 0 & 0 & 1 \\
1 & 0 & 0 & 0 \\
0 & 1 & 0 & 0
\end{array}\right], \quad \Phi_{j, 1}=\left[\begin{array}{cccc}
0 & 0 & -1 & 0 \\
0 & 0 & 0 & 1 \\
1 & 0 & 0 & 0 \\
0 & -1 & 0 & 0
\end{array}\right], \\
& \Phi_{j, i}=\left[\begin{array}{llll}
0 & 0 & 0 & 1 \\
0 & 0 & 1 & 0 \\
0 & 1 & 0 & 0 \\
1 & 0 & 0 & 0
\end{array}\right], \quad \Phi_{j, j}=\left[\begin{array}{cccc}
1 & 0 & 0 & 0 \\
0 & -1 & 0 & 0 \\
0 & 0 & 1 & 0 \\
0 & 0 & 0 & -1
\end{array}\right], \quad \Phi_{j, k}=\left[\begin{array}{cccc}
0 & -1 & 0 & 0 \\
-1 & 0 & 0 & 0 \\
0 & 0 & 0 & 1 \\
0 & 0 & 1 & 0
\end{array}\right], \\
& \Phi_{k, 1}=\left[\begin{array}{cccc}
0 & 0 & 0 & -1 \\
0 & 0 & -1 & 0 \\
0 & 1 & 0 & 0 \\
1 & 0 & 0 & 0
\end{array}\right], \quad \Phi_{k, i}=\left[\begin{array}{cccc}
0 & 0 & -1 & 0 \\
0 & 0 & 0 & 1 \\
-1 & 0 & 0 & 0 \\
0 & 1 & 0 & 0
\end{array}\right], \quad \Phi_{k, j}=\left[\begin{array}{llll}
0 & 1 & 0 & 0 \\
1 & 0 & 0 & 0 \\
0 & 0 & 0 & 1 \\
0 & 0 & 1 & 0
\end{array}\right] \text {, } \\
& \Phi_{k, k}=\left[\begin{array}{cccc}
1 & 0 & 0 & 0 \\
0 & -1 & 0 & 0 \\
0 & 0 & -1 & 0 \\
0 & 0 & 0 & 1
\end{array}\right]
\end{aligned}
$$

Now we can determine some further Clifford algebras as follows.

Recall that $\mathrm{Cl}_{0,0} \cong \mathbb{R}, \mathrm{Cl}_{0,1} \cong \mathbb{C}, \mathrm{Cl}_{1,0} \cong \mathbb{R} \oplus \mathbb{R}, \mathrm{Cl}_{0,2} \cong \mathbb{U}$, and $\mathrm{Cl}_{2,0} \cong \mathbb{R}(2)$. 
By applying isomorphism $\pi_{1}$ to $\mathrm{Cl}_{0,3}$ we have $\mathrm{Cl}_{0,3} \cong \mathrm{Cl}_{1,0} \otimes \mathrm{Cl}_{0,2}$. Since $\mathrm{Cl}_{1,0} \cong \mathbb{R} \oplus$ $\mathbb{R}$ and $\mathrm{Cl}_{0,2} \cong \mathbb{U}$, by Proposition 1.5 (i) we have $\mathrm{Cl}_{0,3} \cong(\mathbb{R} \oplus \mathbb{R}) \otimes \mathbb{U} \cong \mathbb{R} \otimes \mathbb{U} \oplus \mathbb{R} \otimes \mathbb{U} \cong$ $\mathbb{M} \oplus \mathbb{M}$.

Similarly by applying the isomorphism $\pi_{2}$ to $\mathrm{Cl}_{3,0}$ we have $\mathrm{Cl}_{3,0} \cong \mathrm{Cl}_{0,1} \otimes \mathrm{Cl}_{2,0}$. Since $\mathrm{Cl}_{0,1} \cong \mathbb{C}$ and $\mathrm{Cl}_{2,0} \cong \mathbb{R}(2)$, by Proposition 1.5(i) we have $\mathrm{Cl}_{3,0} \cong \mathbb{C} \otimes \mathbb{R}(2) \cong \mathbb{C}(2)$.

By applying $\pi_{1}$ to $\mathrm{Cl}_{0,4}$ we have $\mathrm{Cl}_{0,4} \cong \mathrm{Cl}_{2,0} \otimes \mathrm{Cl}_{0,2}$. Since $\mathrm{Cl}_{2,0} \cong \mathbb{R}(2)$ and $\mathrm{Cl}_{0,2} \cong \mathbb{U}$, by Proposition 1.5(i) we have $\mathrm{Cl}_{0,4} \cong \mathbb{R}(2) \otimes H \cong \mathbb{U}(2)$.

Similarly by applying $\pi_{2}$ to $\mathrm{Cl}_{4,0}$ we have $\mathrm{Cl}_{4,0} \cong \mathrm{Cl}_{0,2} \otimes \mathrm{Cl}_{2,0}$. Since $\mathrm{Cl}_{2,0} \cong \mathbb{R}(2)$ and $\mathrm{Cl}_{0,2} \cong \mathbb{U}$, by Proposition 1.5(i) we have $\mathrm{Cl}_{4,0} \cong \mathbb{R}(2) \otimes \mathbb{U} \cong \mathbb{M}(2)$. If we continue in similar way, we have

$$
\begin{aligned}
& \mathrm{Cl}_{0,5} \cong \mathrm{Cl}_{0,1} \otimes \mathrm{Cl}_{2,0} \otimes \mathrm{Cl}_{0,2} \cong \mathbb{C} \otimes \mathbb{R}(2) \otimes \mathbb{W} \cong \mathbb{C} \otimes(\mathbb{M} \otimes \mathbb{R}(2)) \\
& \cong(\mathbb{C} \otimes \mathbb{U}) \otimes \mathbb{R}(2) \cong \mathbb{C}(2) \otimes \mathbb{R}(2) \cong(\mathbb{C} \otimes \mathbb{R}(2)) \otimes \mathbb{R}(2) \\
& \cong \mathbb{C} \otimes(\mathbb{R}(2) \otimes \mathbb{R}(2)) \cong \mathbb{C} \otimes \mathbb{R}(4) \cong \mathbb{C}(4), \\
& \mathrm{Cl}_{0,6} \cong \mathrm{Cl}_{0,2} \otimes \mathrm{Cl}_{2,0} \otimes \mathrm{Cl}_{0,2} \cong \mathbb{M} \otimes \mathbb{R}(2) \otimes \mathbb{M} \\
& \cong \mathbb{U} \otimes \mathbb{H} \otimes \mathbb{R}(2) \cong \mathbb{R}(4) \otimes \mathbb{R}(2) \cong \mathbb{R}(8), \\
& \mathrm{Cl}_{0,7} \cong \mathrm{Cl}_{0,3} \otimes \mathrm{Cl}_{2,0} \otimes \mathrm{Cl}_{0,2} \cong(\mathbb{H} \oplus \mathbb{U}) \otimes \mathbb{R}(2) \otimes \mathbb{U} \\
& \cong(\mathbb{H} \oplus \mathbb{W}) \otimes \mathbb{H} \otimes \mathbb{R}(2) \cong(\mathbb{H} \otimes \mathbb{H} \oplus \mathbb{H} \otimes \mathbb{U}) \otimes \mathbb{R}(2) \\
& \cong(\mathbb{R}(4) \oplus \mathbb{R}(4)) \otimes \mathbb{R}(2) \cong \mathbb{R}(8) \oplus \mathbb{R}(8), \\
& \mathrm{Cl}_{0,8} \cong \mathrm{Cl}_{0,4} \otimes \mathrm{Cl}_{2,0} \otimes \mathrm{Cl}_{0,2} \cong \mathrm{Cl}_{2,0} \otimes \mathrm{Cl}_{0,2} \otimes \mathrm{Cl}_{2,0} \otimes \mathrm{Cl}_{0,2} \\
& \cong \mathbb{R}(2) \otimes \mathbb{W} \otimes \mathbb{R}(2) \otimes \mathbb{W} \cong \mathbb{R}(2) \otimes \mathbb{R}(2) \otimes \mathbb{W} \otimes \mathbb{W} \cong \mathbb{R}(4) \otimes \mathbb{R}(4) \cong \mathbb{R}(16), \\
& \mathrm{Cl}_{5,0} \cong \mathrm{Cl}_{1,0} \otimes \mathrm{Cl}_{0,2} \otimes \mathrm{Cl}_{2,0} \cong(\mathbb{R} \oplus \mathbb{R}) \otimes \mathbb{H} \otimes \mathbb{R}(2) \\
& \cong(\mathbb{R} \oplus \mathbb{R}) \otimes(\mathbb{R}(2) \otimes \mathbb{W}) \cong(\mathbb{R}(2) \oplus \mathbb{R}(2)) \otimes \mathbb{W} \\
& \cong(\mathbb{R}(2) \otimes \mathbb{W}) \otimes(\mathbb{R}(2) \otimes \mathbb{U}) \cong(\mathbb{M} \otimes \mathbb{R}(2)) \otimes(\mathbb{H} \otimes \mathbb{R}(2)) \\
& \cong \mathbb{H}(2) \oplus \mathbb{H}(2), \\
& \mathrm{Cl}_{6,0} \cong \mathrm{Cl}_{2,0} \otimes \mathrm{Cl}_{0,2} \otimes \mathrm{Cl}_{2,0} \cong \mathbb{R}(2) \otimes \mathbb{H} \otimes \mathbb{R}(2) \cong \mathbb{U} \otimes \mathbb{R}(2) \otimes \mathbb{R}(2) \\
& \cong \mathbb{H} \otimes \mathbb{R}(4) \cong \mathbb{H}(4), \\
& \mathrm{Cl}_{7,0} \cong \mathrm{Cl}_{3,0} \otimes \mathrm{Cl}_{0,2} \otimes \mathrm{Cl}_{2,0} \cong \mathbb{C}(2) \otimes \mathbb{H} \otimes \mathbb{R}(2) \cong \mathbb{C} \otimes \mathbb{R}(2) \otimes \mathbb{H} \otimes \mathbb{R}(2) \\
& \cong \mathbb{C} \otimes \mathbb{M} \otimes \mathbb{R}(2) \otimes \mathbb{R}(2) \cong \mathbb{C}(2) \otimes \mathbb{R}(4) \cong \mathbb{C} \otimes \mathbb{R}(2) \otimes \mathbb{R}(4) \\
& \cong \mathbb{C} \otimes \mathbb{R}(8) \cong \mathbb{C}(8) \text {, } \\
& \mathrm{Cl}_{8,0} \cong \mathrm{Cl}_{4,0} \otimes \mathrm{Cl}_{0,2} \otimes \mathrm{Cl}_{2,0} \cong \mathbb{H}(2) \otimes \mathbb{W} \otimes \mathbb{R}(2) \cong \mathbb{U} \otimes \mathbb{R}(2) \otimes \mathbb{H} \otimes \mathbb{R}(2) \\
& \cong \mathbb{U} \otimes \mathbb{H} \otimes \mathbb{R}(2) \otimes \mathbb{R}(2) \cong \mathbb{R}(4) \otimes \mathbb{R}(4) \cong \mathbb{R}(16)
\end{aligned}
$$


Table 1.1

\begin{tabular}{l|ccccccccc}
\hline$m$ & 0 & 1 & 2 & 3 & 4 & 5 & 6 & 7 & 8 \\
\hline $\mathrm{Cl}_{0, m}$ & $\mathbb{R}$ & $\mathbb{C}$ & $\mathbb{U}$ & $\mathbb{U} \oplus \mathbb{M}$ & $\mathbb{M}(2)$ & $\mathbb{C}(4)$ & $\mathbb{R}(8)$ & $\mathbb{R}(8) \oplus \mathbb{R}(8)$ & $\mathbb{R}(16)$ \\
$\mathrm{Cl}_{m, 0}$ & $\mathbb{R}$ & $\mathbb{R} \oplus \mathbb{R}$ & $\mathbb{R}(2)$ & $\mathbb{C}(2)$ & $\mathbb{M}(2)$ & $\mathbb{M}(2) \oplus \mathbb{U}(2)$ & $\mathbb{H}(4)$ & $\mathbb{C}(8)$ & $\mathbb{R}(16)$ \\
\hline
\end{tabular}

Table 1.2

\begin{tabular}{c|l}
\hline$-q(\bmod 8)$ & $\mathrm{Cl}_{0, q}$ \\
\hline 0,2 & $\mathbb{R}\left(2^{q / 2}\right)$ \\
1 & $\mathbb{R}\left(2^{(q-1) / 2}\right) \oplus \mathbb{R}\left(2^{(q-1) / 2}\right)$ \\
3,7 & $\mathbb{C}\left(2^{(q-1) / 2}\right)$ \\
4,6 & $\mathbb{U}\left(2^{(q-2) / 2}\right)$ \\
5 & $\mathbb{U}\left(2^{(q-1) / 2}\right) \oplus \mathbb{M}\left(2^{(q-1) / 2}\right)$ \\
\hline
\end{tabular}

All of these calculations yields Table 1.1.

By composing the isomorphisms $\pi_{1}$ and $\pi_{2}$ we get an isomorphism from the Clifford algebra $\mathrm{Cl}_{0, m+4}$ to $\mathrm{Cl}_{0, m} \otimes \mathrm{Cl}_{2,0} \otimes \mathrm{Cl}_{0,2}$ as follows:

$$
\begin{aligned}
& \pi: \mathrm{Cl}_{0, m+4} \longrightarrow \mathrm{Cl}_{m+2,0} \otimes \mathrm{Cl}_{0,2} \longrightarrow \mathrm{Cl}_{0, m} \otimes \mathrm{Cl}_{2,0} \otimes \mathrm{Cl}_{0,2}, \\
& \varepsilon_{1} \longmapsto 1 \otimes \varepsilon_{1} \longmapsto 1 \otimes 1 \otimes \varepsilon_{1}, \\
& \varepsilon_{2} \longmapsto 1 \otimes \varepsilon_{2} \longmapsto 1 \otimes 1 \otimes \varepsilon_{2}, \\
& \varepsilon_{3} \longmapsto e_{1} \otimes \varepsilon_{1} \varepsilon_{2} \longmapsto 1 \otimes e_{1} \otimes \varepsilon_{1} \varepsilon_{2}, \\
& \varepsilon_{4} \longmapsto e_{2} \otimes \varepsilon_{1} \varepsilon_{2} \longmapsto 1 \otimes e_{2} \otimes \varepsilon_{1} \varepsilon_{2}, \\
& \varepsilon_{5} \longmapsto e_{3} \otimes \varepsilon_{1} \varepsilon_{2} \longmapsto \varepsilon_{1} \otimes e_{1} e_{2} \otimes \varepsilon_{1} \varepsilon_{2}, \\
& \varepsilon_{6} \longmapsto e_{4} \otimes \varepsilon_{1} \varepsilon_{2} \longmapsto \varepsilon_{2} \otimes e_{1} e_{2} \otimes \varepsilon_{1} \varepsilon_{2}, \\
& \vdots \longmapsto \\
& \vdots \\
& \varepsilon_{n+4} \longmapsto e_{n+2} \otimes \varepsilon_{1} \varepsilon_{2} \longmapsto \varepsilon_{n} \otimes e_{1} e_{2} \otimes \varepsilon_{1} \varepsilon_{2} .
\end{aligned}
$$

In particular, if we take $m=8$ and use the isomorphism $\pi$ two times, then we can write

$$
\mathrm{Cl}_{0,8} \cong \mathrm{Cl}_{2,0} \otimes \mathrm{Cl}_{0,2} \otimes \mathrm{Cl}_{2,0} \otimes \mathrm{Cl}_{0,2}
$$

On the other hand, if we start with the Clifford algebra $\mathrm{Cl}_{0, m+8}$ and apply the isomorphism $\pi$ two times, then we get the isomorphism $\mathrm{Cl}_{0, m+8} \cong \mathrm{Cl}_{0, m} \otimes \mathrm{Cl}_{2,0} \otimes \mathrm{Cl}_{0,2} \otimes \mathrm{Cl}_{2,0} \otimes \mathrm{Cl}_{0,2}$. If use (1.13) in the last expression, then we get the periodicity relation

$$
\mathrm{Cl}_{0, m+8} \cong \mathrm{Cl}_{0, m} \otimes \mathrm{Cl}_{0,8} \cong \mathrm{Cl}_{0, m} \otimes \mathbb{R}(16)
$$

The periodicity $\mathrm{Cl}_{m+8,0} \cong \mathrm{Cl}_{m, 0} \otimes \mathrm{Cl}_{8,0} \cong \mathrm{Cl}_{m, 0} \otimes \mathbb{R}(16)$ can be obtained similarly.

By using the above periodicity relations we can easily determine the Clifford algebras $\mathrm{Cl}_{0, m}$ and $\mathrm{Cl}_{m, 0}$ recursively for the higher values of $m$ and we get Tables 1.2 and 1.3. 
Table 1.3

\begin{tabular}{c|l}
\hline$p(\bmod 8)$ & $\mathrm{Cl}_{p, 0}$ \\
\hline 0,2 & $\mathbb{R}\left(2^{p / 2}\right)$ \\
1 & $\mathbb{R}\left(2^{(p-1) / 2}\right) \oplus \mathbb{R}\left(2^{(p-1) / 2}\right)$ \\
3,7 & $\mathbb{C}\left(2^{(p-1) / 2}\right)$ \\
4,6 & $\mathbb{W}\left(2^{(p-2) / 2}\right)$ \\
5 & $\mathbb{W}\left(2^{(p-1) / 2}\right) \oplus \mathbb{M}\left(2^{(p-1) / 2}\right)$ \\
\hline
\end{tabular}

Table 1.4

\begin{tabular}{c|c|l}
\hline$(p-q)(\bmod 8)$ & $p+q$ & $\mathrm{Cl}_{p, q}$ \\
\hline 0,2 & $2 m$ & $\mathbb{R}\left(2^{m}\right)$ \\
1 & $2 m+1$ & $\mathbb{R}\left(2^{m}\right) \oplus \mathbb{R}\left(2^{m}\right)$ \\
3,7 & $2 m+1$ & $\mathbb{C}\left(2^{m}\right)$ \\
4,6 & $2 m+2$ & $\mathbb{U}\left(2^{m}\right)$ \\
5 & $2 m+3$ & $\mathbb{U}\left(2^{m}\right) \oplus \mathbb{U}\left(2^{m}\right)$ \\
\hline
\end{tabular}

To determine Clifford algebras of type $\mathrm{Cl}_{p, q}(p, q>0)$, the isomorphism $\pi_{3}$ and Tables 1.2 and 1.3 are enough. For example, if we start by applying isomorphism $\pi_{3}$ to $\mathrm{Cl}_{2,2}$, we have $\mathrm{Cl}_{2,2} \cong \mathrm{Cl}_{1,1} \otimes \mathrm{Cl}_{1,1}$. Since $\mathrm{Cl}_{1,1} \cong \mathbb{R}(2)$, by Proposition 1.5(ii) we have $\mathrm{Cl}_{2,2} \cong$ $\mathbb{R}(2) \otimes \mathbb{R}(2) \cong \mathbb{R}(4)$. Similarly $\mathrm{Cl}_{3,3} \cong \mathrm{Cl}_{2,2} \otimes \mathrm{Cl}_{1,1} \cong \mathbb{R}(4) \otimes \mathbb{R}(2) \cong \mathbb{R}(8)$. Similarly for $p=$ $q$ we have $\mathrm{Cl}_{p, p} \cong \mathbb{R}\left(2^{p}\right)$. If we apply $\pi_{3}$ to $\mathrm{Cl}_{1,2}$, then we get $\mathrm{Cl}_{1,2} \cong \mathrm{Cl}_{0,1} \otimes \mathrm{Cl}_{1,1}$. Since $\mathrm{Cl}_{0,1} \cong \mathbb{C}$ and $\mathrm{Cl}_{1,1} \cong \mathbb{R}(2)$, by Proposition 1.5(i) we can write $\mathrm{Cl}_{1,2} \cong \mathbb{C} \otimes \mathbb{R}(2) \cong \mathbb{C}(2)$. Similarly $\mathrm{Cl}_{2,3} \cong \mathrm{Cl}_{1,2} \otimes \mathrm{Cl}_{1,1} \cong \mathbb{C}(2) \otimes \mathbb{R}(2) \cong \mathbb{C}(4)$. Similarly for $q=p+1$ we have $\mathrm{Cl}_{p, p+1} \cong$ $\mathbb{C}\left(2^{p}\right)$. Therefore, by continuing in a completely similar fashion for other values of $p, q(p$, $q>0$ ), we obtain Table 1.4 .

We also point out that there are periodicity isomorphisms $\mathrm{Cl}_{p+8, q} \cong \mathrm{Cl}_{p, q} \otimes \mathrm{Cl}_{8,0}$ and $\mathrm{Cl}_{p, q+8} \cong \mathrm{Cl}_{p, q} \otimes \mathrm{Cl}_{0,8}$ for Clifford algebras (see [4]).

Our goal is give a method for the explicit expressions of the Clifford algebra isomorphisms. To do this firstly we obtain isomorphisms for the Clifford algebras of type $\mathrm{Cl}_{0, m}$.

\section{Isomorphisms of nondegenerate Clifford algebras}

2.1. Isomorphisms for the Clifford algebra $\mathrm{Cl}_{0, m}$. First we obtain isomorphisms of $\mathrm{Cl}_{0, m}$ for $1 \leq m \leq 8$, then by using the periodicity isomorphism $\mathrm{Cl}_{0, m+8} \cong \mathrm{Cl}_{0, m} \otimes \mathrm{Cl}_{0,8} \cong$ $\mathrm{Cl}_{0, m} \otimes \mathbb{R}(16)$ we achieve the other isomorphisms.

2.1.1. Isomorphisms of $\mathrm{Cl}_{0, m}$ for $1 \leq m \leq 8$. Above we have given the isomorphisms $\Psi_{1,0}$ : $\mathrm{Cl}_{0,1} \rightarrow \mathbb{C}$ and $\Psi_{0,2}: \mathrm{Cl}_{0,2} \rightarrow \mathbb{U}$ and the others are as follows. 
8 Explicit isomorphisms of real Clifford algebras

(1) $\Psi_{0,3}: \mathrm{Cl}_{0,3} \rightarrow \mathbb{H} \oplus \mathbb{W}$,

$$
\begin{gathered}
\mathrm{Cl}_{0,3} \longrightarrow \mathrm{Cl}_{1,0} \otimes \mathrm{Cl}_{0,2} \longrightarrow(\mathbb{R} \oplus \mathbb{R}) \otimes \mathbb{H} \longrightarrow \mathbb{H} \oplus \mathbb{H}, \\
\varepsilon_{1} \longmapsto 1 \otimes \varepsilon_{1} \longmapsto(1,1) \otimes i \longmapsto(i, i), \\
\varepsilon_{2} \longmapsto 1 \otimes \varepsilon_{2} \longmapsto(1,1) \otimes j \longmapsto(j, j), \\
\varepsilon_{3} \longmapsto e_{1} \otimes \varepsilon_{1} \varepsilon_{2} \longmapsto(1,-1) \otimes k \longmapsto(k,-k) .
\end{gathered}
$$

(2) $\Psi_{0,4}: \mathrm{Cl}_{0,4} \rightarrow \mathbb{H}(2)$,

$$
\begin{gathered}
\mathrm{Cl}_{0,4} \longrightarrow \mathrm{Cl}_{2,0} \otimes \mathrm{Cl}_{0,2} \longrightarrow \mathbb{R}(2) \otimes \mathbb{U} \longrightarrow \mathbb{\mathbb { }}(2), \\
\varepsilon_{1} \longmapsto 1 \otimes \varepsilon_{1} \longmapsto\left[\begin{array}{ll}
1 & 0 \\
0 & 1
\end{array}\right] \otimes i \longmapsto\left[\begin{array}{ll}
i & 0 \\
0 & i
\end{array}\right], \\
\varepsilon_{2} \longmapsto 1 \otimes \varepsilon_{2} \longmapsto\left[\begin{array}{cc}
1 & 0 \\
0 & 1
\end{array}\right] \otimes j \longmapsto\left[\begin{array}{cc}
j & 0 \\
0 & j
\end{array}\right], \\
\varepsilon_{3} \longmapsto e_{1} \otimes \varepsilon_{1} \varepsilon_{2} \longmapsto\left[\begin{array}{cc}
0 & 1 \\
1 & 0
\end{array}\right] \otimes k \longmapsto\left[\begin{array}{cc}
0 & k \\
k & 0
\end{array}\right], \\
\varepsilon_{4} \longmapsto e_{2} \otimes \varepsilon_{1} \varepsilon_{2} \longmapsto\left[\begin{array}{cc}
1 & 0 \\
0 & -1
\end{array}\right] \otimes k \longmapsto\left[\begin{array}{cc}
k & 0 \\
0 & -k
\end{array}\right] .
\end{gathered}
$$

(3) $\Psi_{0,5}: \mathrm{Cl}_{0,5} \rightarrow \mathbb{C}(4)$,

$$
\begin{gathered}
\mathrm{Cl}_{0,5} \longrightarrow \mathbb{C} \otimes \mathbb{R}(4) \longrightarrow \mathbb{C}(4), \\
\varepsilon_{1} \longmapsto i \otimes\left[\begin{array}{cccc}
-1 & 0 & 0 & 0 \\
0 & -1 & 0 & 0 \\
0 & 0 & 1 & 0 \\
0 & 0 & 0 & 1
\end{array}\right] \longmapsto\left[\begin{array}{cccc}
-i & 0 & 0 & 0 \\
0 & -i & 0 & 0 \\
0 & 0 & i & 0 \\
0 & 0 & 0 & i
\end{array}\right], \\
\varepsilon_{2} \longmapsto 1 \otimes\left[\begin{array}{cccc}
0 & 0 & 1 & 0 \\
0 & 0 & 0 & 1 \\
-1 & 0 & 0 & 0 \\
0 & -1 & 0 & 0
\end{array}\right] \longmapsto\left[\begin{array}{cccc}
0 & 0 & 1 & 0 \\
0 & 0 & 0 & 1 \\
-1 & 0 & 0 & 0 \\
0 & -1 & 0 & 0
\end{array}\right],
\end{gathered}
$$




$$
\begin{gathered}
\varepsilon_{3} \longmapsto i \otimes\left[\begin{array}{cccc}
0 & 0 & 0 & -1 \\
0 & 0 & -1 & 0 \\
0 & -1 & 0 & 0 \\
-1 & 0 & 0 & 0
\end{array}\right] \\
\varepsilon_{4} \longmapsto i \otimes\left[\begin{array}{cccc}
0 & 0 & -1 & 0 \\
0 & 0 & 0 & 1 \\
-1 & 0 & 0 & 0 \\
0 & 1 & 0 & 0
\end{array}\right] \longmapsto\left[\begin{array}{cccc}
0 & 0 & 0 & -i \\
0 & 0 & -i & 0 \\
0 & -i & 0 & 0 \\
-i & 0 & 0 & 0
\end{array}\right], \\
\varepsilon_{5} \longmapsto 1 \otimes\left[\begin{array}{cccc}
0 & 0 & -i & 0 \\
0 & 0 & 0 & i \\
-i & 0 & 0 & 0 \\
0 & i & 0 & 0
\end{array}\right], \\
\left.0 \begin{array}{cccc}
0 & 0 & 0 & -1 \\
0 & 0 & 1 & 0 \\
0 & -1 & 0 & 0 \\
1 & 0 & 0 & 0
\end{array}\right]
\end{gathered}
$$

(4) $\Psi_{0,6}: \mathrm{Cl}_{0,6} \rightarrow \mathbb{R}(8)$,

$$
\begin{aligned}
& \Psi_{0,6}\left(\varepsilon_{1}\right)=-\sigma_{2} \otimes \sigma_{1} \sigma_{2} \otimes I, \\
& \Psi_{0,6}\left(\varepsilon_{2}\right)=-\sigma_{1} \sigma_{2} \otimes I \otimes I, \\
& \Psi_{0,6}\left(\varepsilon_{3}\right)=-\sigma_{1} \otimes \sigma_{1} \sigma_{2} \otimes \sigma_{1}, \\
& \Psi_{0,6}\left(\varepsilon_{4}\right)=-\sigma_{1} \otimes \sigma_{1} \sigma_{2} \otimes \sigma_{2}, \\
& \Psi_{0,6}\left(\varepsilon_{5}\right)=\sigma_{1} \otimes I \otimes \sigma_{1} \sigma_{2}, \\
& \Psi_{0,6}\left(\varepsilon_{6}\right)=-\sigma_{2} \otimes \sigma_{1} \otimes \sigma_{1} \sigma_{2},
\end{aligned}
$$

where $I=\left[\begin{array}{ll}1 & 0 \\ 0 & 1\end{array}\right], \sigma_{1}=\left[\begin{array}{ll}0 & 1 \\ 1 & 0\end{array}\right]$, and $\sigma_{2}=\left[\begin{array}{cc}1 & 0 \\ 0 & -1\end{array}\right]$.

(5) $\Psi_{0,7}: \mathrm{Cl}_{0,6} \rightarrow \mathbb{R}(8) \oplus \mathbb{R}(8)$,

$$
\begin{gathered}
\varepsilon_{1} \longmapsto\left(-\sigma_{2} \otimes \sigma_{1} \sigma_{2} \otimes I,-\sigma_{2} \otimes \sigma_{1} \sigma_{2} \otimes I\right), \\
\mathcal{E}_{2} \longmapsto\left(-\sigma_{1} \sigma_{2} \otimes I \otimes I,-\sigma_{1} \sigma_{2} \otimes I \otimes I\right), \\
\mathcal{E}_{3} \longmapsto\left(-\sigma_{1} \otimes \sigma_{1} \sigma_{2} \otimes \sigma_{1},-\sigma_{1} \otimes \sigma_{1} \sigma_{2} \otimes \sigma_{1}\right), \\
\mathcal{E}_{4} \longmapsto\left(-\sigma_{1} \otimes \sigma_{1} \sigma_{2} \otimes \sigma_{2},-\sigma_{1} \otimes \sigma_{1} \sigma_{2} \otimes \sigma_{2}\right), \\
\varepsilon_{5} \longmapsto\left(\sigma_{1} \otimes I \otimes \sigma_{1} \sigma_{2}, \sigma_{1} \otimes I \otimes \sigma_{1} \sigma_{2}\right), \\
\mathcal{E}_{6} \longmapsto\left(-\sigma_{2} \otimes \sigma_{1} \otimes \sigma_{1} \sigma_{2},-\sigma_{2} \otimes \sigma_{1} \otimes \sigma_{1} \sigma_{2}\right), \\
\mathcal{E}_{7} \longmapsto\left(\sigma_{2} \otimes \sigma_{2} \otimes \sigma_{1} \sigma_{2}, \sigma_{2} \otimes \sigma_{2} \otimes \sigma_{1} \sigma_{2}\right),
\end{gathered}
$$


10 Explicit isomorphisms of real Clifford algebras

(6) $\Psi_{0,8}: \mathrm{Cl}_{0,8} \rightarrow \mathbb{R}(16)$,

$$
\begin{aligned}
& \varepsilon_{1} \longmapsto-I \otimes I \otimes \sigma_{2} \otimes \sigma_{1} \sigma_{2}, \\
& \varepsilon_{2} \longmapsto-I \otimes I \otimes \sigma_{1} \sigma_{2} \otimes I, \\
& \varepsilon_{3} \longmapsto-I \otimes \sigma_{1} \otimes \sigma_{1} \otimes \sigma_{1} \sigma_{2}, \\
& \varepsilon_{4} \longmapsto-I \otimes \sigma_{2} \otimes \sigma_{1} \otimes \sigma_{1} \sigma_{2}, \\
& \mathcal{E}_{5} \longmapsto I \otimes \sigma_{1} \sigma_{2} \otimes \sigma_{1} \otimes I, \\
& \mathcal{E}_{6} \longmapsto-I \otimes \sigma_{1} \sigma_{2} \otimes \sigma_{2} \otimes \sigma_{1}, \\
& \mathcal{E}_{7} \longmapsto \sigma_{1} \otimes \sigma_{1} \sigma_{2} \otimes \sigma_{2} \otimes \sigma_{2}, \\
& \varepsilon_{8} \longmapsto \sigma_{2} \otimes \sigma_{1} \sigma_{2} \otimes \sigma_{2} \otimes \sigma_{2} .
\end{aligned}
$$

2.1.2. Isomorphisms of $\mathrm{Cl}_{0, n+8}$ for $n \geq 1$. Now we want to obtain explicit form of the isomorphism (2):

$$
\begin{gathered}
\mathrm{Cl}_{0, n+8} \longrightarrow \mathrm{Cl}_{0, n+4} \otimes \mathrm{Cl}_{2,0} \otimes \mathrm{Cl}_{0,2} \longrightarrow \mathrm{Cl}_{0, n} \otimes \mathrm{Cl}_{2,0} \otimes \mathrm{Cl}_{0,2} \otimes \mathrm{Cl}_{2,0} \otimes \mathrm{Cl}_{0,2}, \\
\varepsilon_{1} \longmapsto 1 \otimes 1 \otimes \varepsilon_{1} \longmapsto 1 \otimes 1 \otimes 1 \otimes 1 \otimes \varepsilon_{1}, \\
\varepsilon_{2} \longmapsto 1 \otimes 1 \otimes \varepsilon_{2} \longmapsto 1 \otimes 1 \otimes 1 \otimes 1 \otimes \varepsilon_{2}, \\
\varepsilon_{3} \longmapsto 1 \otimes e_{1} \otimes \varepsilon_{1} \varepsilon_{2} \longmapsto 1 \otimes 1 \otimes 1 \otimes e_{1} \otimes \varepsilon_{1} \varepsilon_{2}, \\
\varepsilon_{4} \longmapsto 1 \otimes e_{2} \otimes \varepsilon_{1} \varepsilon_{2} \longmapsto 1 \otimes 1 \otimes 1 \otimes e_{2} \otimes \varepsilon_{1} \varepsilon_{2}, \\
\varepsilon_{5} \longmapsto \varepsilon_{1} \otimes e_{1} e_{2} \otimes \varepsilon_{1} \varepsilon_{2} \longmapsto 1 \otimes 1 \otimes \varepsilon_{1} \otimes e_{1} e_{2} \otimes \varepsilon_{1} \varepsilon_{2}, \\
\varepsilon_{6} \longmapsto \varepsilon_{2} \otimes e_{1} e_{2} \otimes \varepsilon_{1} \varepsilon_{2} \longmapsto 1 \otimes 1 \otimes \varepsilon_{2} \otimes e_{1} e_{2} \otimes \varepsilon_{1} \varepsilon_{2}, \\
\mathcal{E}_{7} \longmapsto \varepsilon_{3} \otimes e_{1} e_{2} \otimes \varepsilon_{1} \varepsilon_{2} \longmapsto 1 \otimes e_{1} \otimes \varepsilon_{1} \varepsilon_{2} \otimes e_{1} e_{2} \otimes \varepsilon_{1} \varepsilon_{2}, \\
\varepsilon_{8} \longmapsto \varepsilon_{4} \otimes e_{1} e_{2} \otimes \varepsilon_{1} \varepsilon_{2} \longmapsto 1 \otimes e_{2} \otimes \varepsilon_{1} \varepsilon_{2} \otimes e_{1} e_{2} \otimes \varepsilon_{1} \varepsilon_{2}, \\
\varepsilon_{9} \longmapsto \varepsilon_{5} \otimes e_{1} e_{2} \otimes \varepsilon_{1} \varepsilon_{2} \longmapsto \varepsilon_{1} \otimes e_{1} e_{2} \otimes \varepsilon_{1} \varepsilon_{2} \otimes e_{1} e_{2} \otimes \varepsilon_{1} \varepsilon_{2}, \\
\varepsilon_{10} \longmapsto \varepsilon_{6} \otimes e_{1} e_{2} \otimes \varepsilon_{1} \varepsilon_{2} \longmapsto \varepsilon_{2} \otimes e_{1} e_{2} \otimes \varepsilon_{1} \varepsilon_{2} \otimes e_{1} e_{2} \otimes \varepsilon_{1} \varepsilon_{2}, \\
\vdots \\
\varepsilon_{n+8} \longmapsto \varepsilon_{n+4} \otimes e_{1} e_{2} \otimes \varepsilon_{1} \varepsilon_{2} \longmapsto \varepsilon_{n} \otimes e_{1} e_{2} \otimes \varepsilon_{1} \varepsilon_{2} \otimes e_{1} e_{2} \otimes \varepsilon_{1} \varepsilon_{2} .
\end{gathered}
$$


Then we get the isomorphism $\Psi_{0, n+8}$ as

$$
\begin{aligned}
\mathrm{Cl}_{0, n+8} & \longrightarrow \mathrm{Cl}_{0, n} \otimes \mathbb{R}(16), \\
\varepsilon_{1} & \longmapsto 1 \otimes A_{1}, \\
\varepsilon_{2} & \longmapsto 1 \otimes A_{2}, \\
\varepsilon_{3} & \longmapsto 1 \otimes A_{3}, \\
\varepsilon_{4} & \longmapsto 1 \otimes A_{4}, \\
\varepsilon_{5} & \longmapsto 1 \otimes A_{5}, \\
\varepsilon_{6} & \longmapsto 1 \otimes A_{6}, \\
\varepsilon_{7} & \longmapsto 1 \otimes A_{7}, \\
\varepsilon_{8} & \longmapsto 1 \otimes A_{8}, \\
\varepsilon_{9} & \longmapsto \varepsilon_{1} \otimes B, \\
\varepsilon_{10} & \longmapsto \varepsilon_{2} \otimes B, \\
\vdots & \vdots \\
\varepsilon_{n+8} & \longmapsto \varepsilon_{n} \otimes B,
\end{aligned}
$$

where $A_{1}=\Psi_{0,8}\left(\varepsilon_{1}\right), A_{2}=\Psi_{0,8}\left(\varepsilon_{2}\right), A_{3}=\Psi_{0,8}\left(\varepsilon_{3}\right), A_{4}=\Psi_{0,8}\left(\varepsilon_{4}\right), A_{5}=\Psi_{0,8}\left(\varepsilon_{5}\right), A_{6}=$ $\Psi_{0,8}\left(\varepsilon_{6}\right), A_{7}=\Psi_{0,8}\left(\varepsilon_{7}\right), A_{8}=\Psi_{0,8}\left(\varepsilon_{8}\right)$ and $B=\sigma_{1} \sigma_{2} \otimes \sigma_{1} \sigma_{2} \otimes \sigma_{2} \otimes \sigma_{2}$. Note that $B$ is symmetric with $B^{2}=I$ and it anticommutes with the matrices $A_{1}, A_{2}, \ldots, A_{8}$. The explicit form $B$ is as follows:

$$
B=\left[\begin{array}{cccccccccccccccc}
0 & 0 & 0 & 0 & 0 & 0 & 0 & 0 & 0 & 0 & 0 & 0 & 1 & 0 & 0 & 0 \\
0 & 0 & 0 & 0 & 0 & 0 & 0 & 0 & 0 & 0 & 0 & 0 & 0 & -1 & 0 & 0 \\
0 & 0 & 0 & 0 & 0 & 0 & 0 & 0 & 0 & 0 & 0 & 0 & 0 & 0 & -1 & 0 \\
0 & 0 & 0 & 0 & 0 & 0 & 0 & 0 & 0 & 0 & 0 & 0 & 0 & 0 & 0 & 1 \\
0 & 0 & 0 & 0 & 0 & 0 & 0 & 0 & -1 & 0 & 0 & 0 & 0 & 0 & 0 & 0 \\
0 & 0 & 0 & 0 & 0 & 0 & 0 & 0 & 0 & 1 & 0 & 0 & 0 & 0 & 0 & 0 \\
0 & 0 & 0 & 0 & 0 & 0 & 0 & 0 & 0 & 0 & 1 & 0 & 0 & 0 & 0 & 0 \\
0 & 0 & 0 & 0 & 0 & 0 & 0 & 0 & 0 & 0 & 0 & -1 & 0 & 0 & 0 & 0 \\
0 & 0 & 0 & 0 & -1 & 0 & 0 & 0 & 0 & 0 & 0 & 0 & 0 & 0 & 0 & 0 \\
0 & 0 & 0 & 0 & 0 & 1 & 0 & 0 & 0 & 0 & 0 & 0 & 0 & 0 & 0 & 0 \\
0 & 0 & 0 & 0 & 0 & 0 & 1 & 0 & 0 & 0 & 0 & 0 & 0 & 0 & 0 & 0 \\
0 & 0 & 0 & 0 & 0 & 0 & 0 & -1 & 0 & 0 & 0 & 0 & 0 & 0 & 0 & 0 \\
1 & 0 & 0 & 0 & 0 & 0 & 0 & 0 & 0 & 0 & 0 & 0 & 0 & 0 & 0 & 0 \\
0 & -1 & 0 & 0 & 0 & 0 & 0 & 0 & 0 & 0 & 0 & 0 & 0 & 0 & 0 & 0 \\
0 & 0 & -1 & 0 & 0 & 0 & 0 & 0 & 0 & 0 & 0 & 0 & 0 & 0 & 0 & 0 \\
0 & 0 & 0 & 1 & 0 & 0 & 0 & 0 & 0 & 0 & 0 & 0 & 0 & 0 & 0 & 0
\end{array}\right] .
$$


2.2. Isomorphisms for the Clifford algebra $\mathrm{Cl}_{m, 0}$. Now by using the above isomorphism we determine isomorphisms for Clifford algebras $\mathrm{Cl}_{m, 0}$. Above we have given the isomorphisms $\Psi_{1,0}: \mathrm{Cl}_{1,0} \rightarrow \mathbb{R} \oplus \mathbb{R}$ and $\Psi_{2,0}: \mathrm{Cl}_{2,0} \rightarrow \mathbb{R}(2)$ and the others can be obtained easily. For example, we know that $\mathrm{Cl}_{3,0} \cong \mathrm{Cl}_{0,1} \otimes \mathrm{Cl}_{2,0}$,

$$
\begin{aligned}
\mathrm{Cl}_{3,0} \longrightarrow \mathrm{Cl}_{0,1} \otimes \mathrm{Cl}_{2,0} \longrightarrow \mathbb{C} \otimes \mathbb{R}(2) \longrightarrow \mathbb{C}(2), \\
e_{1} \longmapsto 1 \otimes e_{1} \longmapsto 1 \otimes \sigma_{1} \longmapsto \sigma_{1}, \\
e_{2} \longmapsto 1 \otimes e_{2} \longmapsto 1 \otimes \sigma_{2} \longmapsto \sigma_{2}, \\
e_{3} \longmapsto \varepsilon_{1} \otimes e_{1} e_{2} \longmapsto i \otimes \sigma_{1} \sigma_{2} \longmapsto i \sigma_{1} \sigma_{2}
\end{aligned}
$$

that is, $\Psi_{3,0}=\Psi_{0,1} \otimes \Psi_{2,0}$. Generally the isomorphism $\Psi_{n+2,0}$ of $\mathrm{Cl}_{n+2,0}$ can be expressed as $\Psi_{n+2,0}=\Psi_{0, n} \otimes \Psi_{2,0}$ since $\mathrm{Cl}_{n+2,0} \cong \mathrm{Cl}_{0, n} \otimes \mathrm{Cl}_{2,0}$.

2.3. Isomorphisms for the Clifford algebra $\mathrm{Cl}_{p, q}(p, q>0)$. Now by using the above isomorphisms we determine isomorphisms for Clifford algebras $\mathrm{Cl}_{p, q}$. Above we have given the isomorphism $\Psi_{1,1}: \mathrm{Cl}_{1,1} \rightarrow \mathbb{R}(2)$ and the others can be obtained easily. For example, we know that $\mathrm{Cl}_{2,2} \cong \mathrm{Cl}_{1,1} \otimes \mathrm{Cl}_{1,1}$,

$$
\begin{aligned}
& \mathrm{Cl}_{2,2} \longrightarrow \mathrm{Cl}_{1,1} \otimes \mathrm{Cl}_{1,1} \longrightarrow \mathbb{R}(2) \otimes \mathbb{R}(2) \longrightarrow \mathbb{R}(4), e_{1} \longmapsto 1 \otimes e_{1} \longmapsto\left[\begin{array}{ll}
1 & 0 \\
0 & 1
\end{array}\right] \otimes\left[\begin{array}{ll}
0 & 1 \\
1 & 0
\end{array}\right] \longmapsto I \otimes \sigma_{1}, \\
& e_{2} \longmapsto e_{1} \otimes e_{1} \varepsilon_{1} \longmapsto\left[\begin{array}{ll}
0 & 1 \\
1 & 0
\end{array}\right] \otimes\left[\begin{array}{cc}
1 & 0 \\
0 & -1
\end{array}\right] \longmapsto \sigma_{1} \otimes \sigma_{2}, \\
& \varepsilon_{1} \longmapsto 1 \otimes \varepsilon_{1} \longmapsto\left[\begin{array}{cc}
1 & 0 \\
0 & 1
\end{array}\right] \otimes\left[\begin{array}{ll}
0 & -1 \\
1 & 0
\end{array}\right] \longmapsto I \otimes \sigma_{1} \sigma_{2}, \\
& \varepsilon_{2} \longmapsto \varepsilon_{1} \otimes e_{1} \varepsilon_{1} \longmapsto\left[\begin{array}{cc}
0 & -1 \\
1 & 0
\end{array}\right] \otimes\left[\begin{array}{cc}
1 & 0 \\
0 & -1
\end{array}\right] \longmapsto \sigma_{1} \sigma_{2} \otimes \sigma_{2},
\end{aligned}
$$

that is, $\Psi_{2,2}=\Psi_{1,1} \otimes \Psi_{1,1}$. Generally the isomorphism $\Psi_{p+1, q+1}$ of $\mathrm{Cl}_{p+1, q+1}$ can be expressed as $\Psi_{p+1, q+1}=\Psi_{p, q} \otimes \Psi_{1,1}$ since $\mathrm{Cl}_{p+1, q+1} \cong \mathrm{Cl}_{p, q} \otimes \mathrm{Cl}_{1,1}$. 


\section{References}

[1] M. Artin, Algebra, Prentice-Hall, New Jersey, 1991.

[2] F. R. Harvey, Spinors and Calibrations, Perspectives in Mathematics, vol. 9, Academic Press, Massachusetts, 1990.

[3] H. B. Lawson Jr. and M.-L. Michelsohn, Spin Geometry, Princeton Mathematical Series, vol. 38, Princeton University Press, New Jersey, 1989.

[4] P. Lounesto, Clifford Algebras and Spinors, London Mathematical Society Lecture Note Series, vol. 239, Cambridge University Press, Cambridge, 1997.

N. Değirmenci: Department of Mathematics, Faculty of Science, Anadolu University, 26470 Eskisehir, Turkey

E-mail address: ndegirmenci@anadolu.edu.tr

Ş. Karapazar: Department of Mathematics, Faculty of Science, Anadolu University, 26470 Eskisehir, Turkey

E-mail address: skarapazar@anadolu.edu.tr 


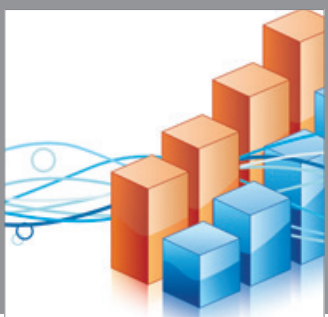

Advances in

Operations Research

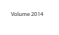

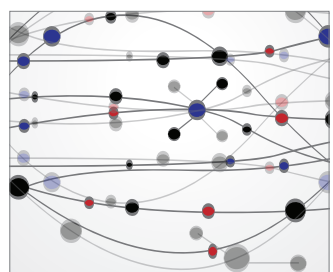

\section{The Scientific} World Journal
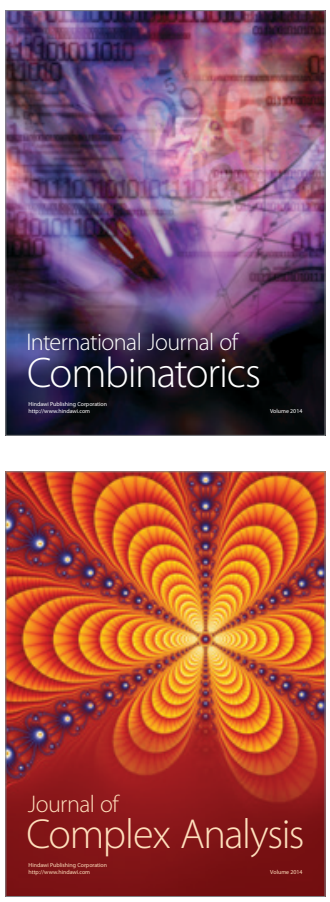

International Journal of

Mathematics and

Mathematical

Sciences
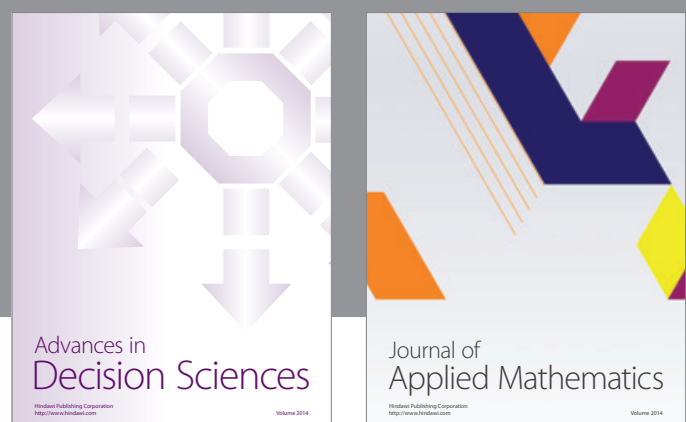

Journal of

Applied Mathematics
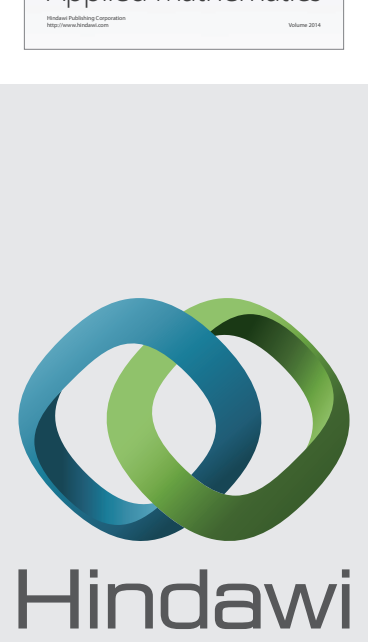

Submit your manuscripts at http://www.hindawi.com
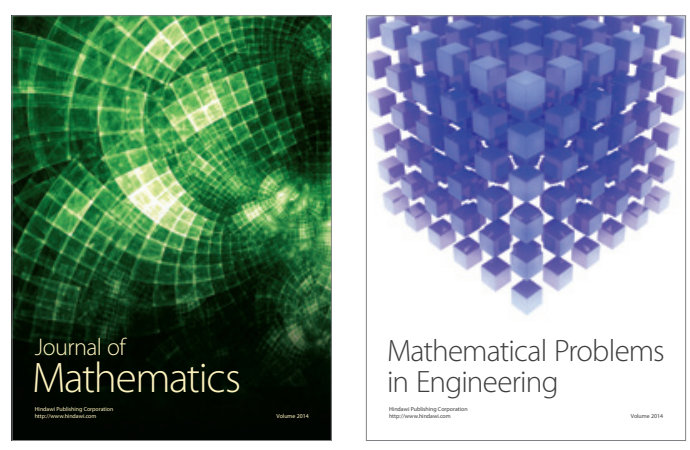

Mathematical Problems in Engineering
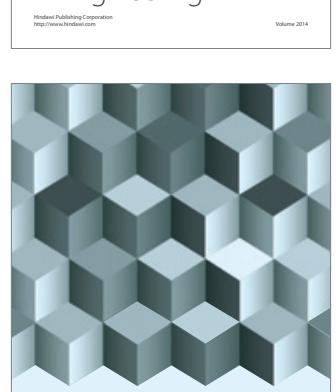

Journal of

Function Spaces
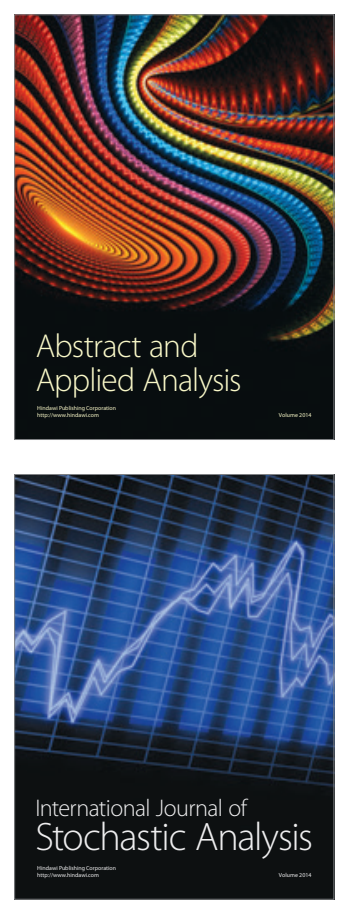

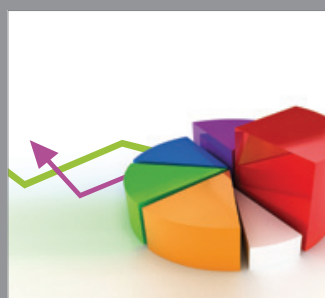

ournal of

Probability and Statistics

Promensencen
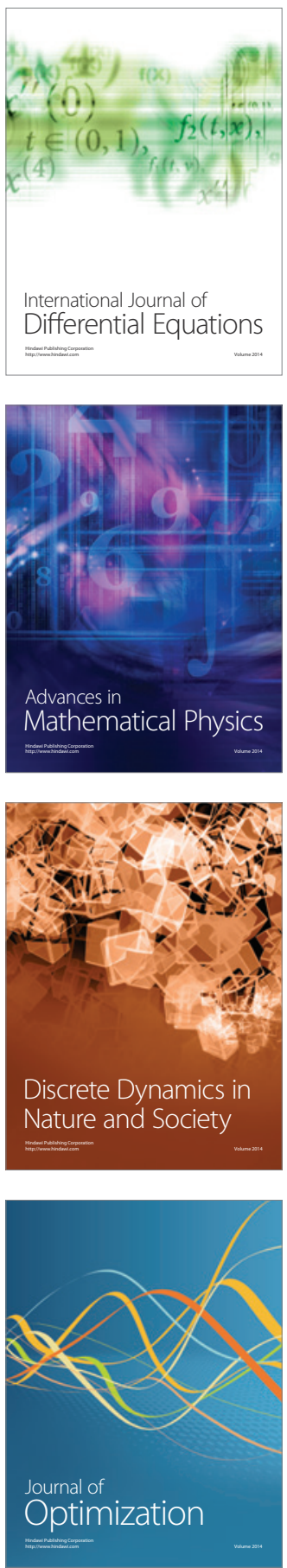